\title{
Some Aspects of the Theory of Defects of Ordered Media and Gauge Fields Related to Foliations
}

\author{
V. Poénaru \\ Université Paris-Sud, Bâtiment mathématique 425, F-91405 Orsay, France
}

\begin{abstract}
When translational symmetry is broken in the ground state, the homotopy theory of defects of ordered media has to be supplemented with integrability conditions, coming from the theory of foliations. These show how some homotopy classes split into several distinct defects, while other homotopy classes do not occur physically. This framework can also be used in order to discuss defects of gauge fields, where in a first approximation classifying spaces play the role of the manifolds of internal states.
\end{abstract}

\section{Introduction}

During the last few years, the topological theory of defects of ordered media has become a relatively well established subject.

The general framework for this approach, as laid down by Toulouse and Kléman, has been, so far, the following. For each type of order, one has a "manifold of internal states", $V$, characteristic for the order in question.

The mathematical model for a specific ordered medium consists of a physical space $M$ (which is assumed to be an $n$-dimensional manifold), of a subset of "defect points" $\Sigma \subset M$, and of a continuously defined "order parameter"

$$
M-\Sigma \ni p \mapsto \Phi(p) \in V .
$$

The standard approach has been to classify defects according to the homotopy classes of the maps $\Phi$ or to study the interaction of defects via the algebraic structure of the homotopy group $\pi_{i} V$ and their Whitehead products (see, for example $[20,7,15,16,22])$.

General references for all this are Mermin's review article [9], our Les Houches lectures [17], or the review article of Michel [10].

Limitations of this approach, in the case of ordered media with broken translational symmetry in the uniform state, are very carefully explained in one of the paragraphs of Mermin's article.

The aim of the present paper is to go one small step beyond pure homotopy theory, towards differential geometry, in this topological study of defects. The new 

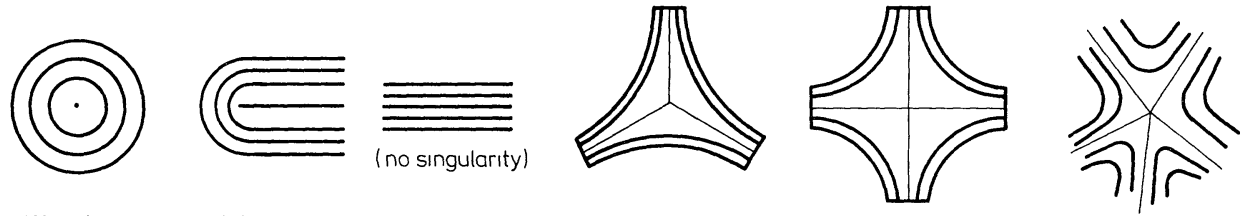

Fig. 1. Measured foliations (2-dimensional "smectics")

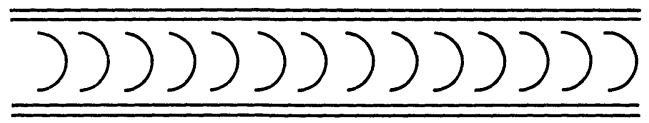

Fig. 2. Non-measured foliation

mathematical tool which we will be putting to use is the "theory of foliations". We will give now a very sketchy idea of what this is all about; more mathematical details are to be found in the appendix at the end of this paper.

Assume, for simplicity, that the physical space $M$ is an open region of the euclidean $n$-space $R^{n}$ and that $V$ is the set of all $k$-dimensional linear subvarieties of $R^{n}$ (this is called the Grassman manifold $G_{n, k}$ ). Assume, also, that the order parameter $\Phi$ associates to every $p \in M-\Sigma$ such a $k$-dimensional linear subvariety $\Phi(p)$ passing through $p$. One will say that $\Phi$ defines a foliation if $M-\Sigma$ can be completely covered by two-by-two disjoint, $k$-dimensional smooth, connected layers such that $\Phi(p)$ is the tangent space of the (unique) layer passing through the point $p$.

If $k=1$, such layers always exist, because one just has to integrate an ordinary differential equation in order to get them. But if $k \geqq 2$, a field of $k$-dimensional planes $\Phi$ very seldom defines a foliation. The condition for this to be the case is a non-linear "integrability condition" involving the first order derivations of the map $\Phi$. If the condition is satisfied, we say that $\Phi$ is "integrable" (and if this is so, then a foliation $\mathscr{F}$ is defined by $\Phi$ ).

A very important class of foliations are the so-called "measured foliations", for which the layers (or rather "leaves" as they are usually called) are all equidistant. One can think of a measured foliation as being a very rough mathematical model of a smectic liquid crystal. Figure 1 below shows some examples of such measured foliations, with singularities, in dimension $2(n=2, k=1)$.

By contrast, the foliation in Fig. 2 is not measured.

Now, with respect to the standard homotopy theory, here comes a new fact. If our ordered medium is modeled by a (measured) foliation with $V=G_{n, k}$, although every individual value $\Phi(p) \in V$ is acceptable, a global map $M-\Sigma \stackrel{\Phi}{\longrightarrow} V$ is not necessarily acceptable. All this is very much in line with Mermin's critique.

The first two paragraphs of this paper will give instances of the following two basic facts (in this framework of ordered media defined by foliations):

(i) Not every (homotopy class of) defect(s) predicted by pure homotopy theory is necessarily realized. In particular, we show that for a punctual defect of a twodimensional smectic the index of the corresponding plane field takes only the 
values :

$$
1, \frac{1}{2}, 0,-\frac{1}{2},-1,-\frac{3}{2},-2, \ldots,
$$

but never values strictly larger that $1^{1}$.

(ii) Two defects which might look homotopically the same need not be continuously deformable into each other if the integrability condition for the map $M-\Sigma \rightarrow V$ is to be respected. In particular, answering a question of Michel, we will exhibit a defect of a foliated (='layered) structure which, homotopically speaking, is trivial (if one forgets about the integrability conditions).

So, in the context of ordered media described by foliations, not only certain homotopy classes of defects are not physically realizable, but moreover, the same homotopy class of defects can split into several physically distinct defect classes.

Of course, two defects which are homotopically distinct are also physically distinct. That much of the homotopy classification stays true, without exception.

Changing the topic, foliations also appear in the context of gauge fields, or from a more mathematical standpoint when one consideres connections and curvature. The last paragraphs of this paper will contain some preliminary remarks about "defects of a gauge field" and about disclinations in crystalline systems, viewed from this angle. We hope to pursue these matters in a subsequent paper. The reader should be warned that this last paragraph requires more mathematical background than the first two, which we have tried to keep as elementary as possible.

Let us finish with a last comment. One of the things in which the homotopy theory of defects has not been very successful, is coping with energy consideration. The extra refinement which foliation theory brings to pure homotopy should, at least partially, be able to bridge the gap and make energy computations accessible by geometric-topological means. A forthcoming paper of Langevin [5] seems to go quite far in this direction; otherwise, his approach is quite different from ours.

\section{Punctual Defects of 2-Dimensional Smectics}

Let $\Phi$ be a smooth field of directors (lines) in $R^{2}$, with an isolated singularity at the origin. It is well known that the following conditions are equivalent:

1) One can put consistent arrows on the directors, or, in other words there is an underlying vector field, to $\Phi$, which we will denote by $\xi$.

2) Considered as a line bundle, $\Phi$ is trivial (i.e. orientable).

3) The orthogonal line bundle $\Phi^{\perp}$ is trivial (i.e. orientable).

4) There is a differential 1 -form $\omega$ with an isolated singularity at the origin, such that $\Phi$ is just the kernel of $\omega$.

If all this is the case, we will call $\Phi$ "orientable".

If $\Phi$ is assumed orientable, the following facts are also equivalent:

(i) The foliation defined by $\Phi$ is measured. (Note that in any case $\Phi$ defines a foliation with an isolated singularity at 0 , since $k=1$. We will call this foliation $\mathscr{F}$. Conversely a 1-dimensional foliation $\mathscr{F}$ defines a field of directors.)

1 We understand that P. Ginsparg and N.D. Mermin have established a similar result (unpublished) 
(ii) The 1 -form $\omega$ is closed (i.e. $d \omega=0$ ).

(iii) $\operatorname{rot} \xi=0$.

(iv) $\operatorname{rot} \xi^{\perp}=0$.

Now, an arbitary $\Phi$ lifts automatically to the ramified 2-sheeted covering of $R^{2}$ (the "Riemann surface" of $\sqrt{z}$ ). This lift is a new field of directors on $R^{2}$ with an isolated singularity at 0 , and we will denote it by $2 \Phi$. It is easy to see that the field $2 \Phi$ is always orientable.

For a vector field $\xi$ with an isolated singularity at 0 , one defines, classically, the index of $\xi: I(\xi) \in Z$ (=the integers), which measures how many times $\xi$ turns around 0 .

The following two facts are easily established:

1) $I(\xi)=I(-\xi)$.

2) $I(2 \xi)=2 I(\xi)-1$.

In view of 1), the index $I(\xi)$ depends only on the director field associated to $\xi$, and in view of 2), we can consistently define the index of an arbitrary field of directors $\Phi$, as being the half integer $I(\Phi)=\frac{I(2 \Phi)+1}{2}$.

Examples. In Fig. 1, the corresponding field is orientable if and only if the "number of prongs", $q$, is even. Moreover, one checks easily that: $I=1-\frac{q}{2}$.

We leave it as an exercise to the reader to construct $\Phi$ 's with an arbitrary halfintegral $I(\Phi)$.

As already said, we will consider measured (possibly non-orientable) 1-dimensional foliations $\mathscr{F}$ in a 2-dimensional physical space as a mathematical model for 2-dimension smectic liquid crystals.

The purpose of this paragraphs is to establish the following:

Theorem 1. Let $\Phi$ be a field of directors in $R^{2}$ with an isolated singularity at 0 , defining a measured foliation. Then $I(\Phi) \leqq 1$. In particular, a vector field $\xi$ on $R^{2}$, with an isolated singularity at 0 , such that rot $\xi=0$, has the property that $I(\xi) \leqq 1$.

We start by noting that $2 \Phi$ also defines a measured foliation and hence it is the kernel of a closed 1-form $\omega$. By the so-called "Poincaré lemma" [3], any closed form in $R^{n}$ is exact, and so the proof of our Theorem 1 is reduced to the following:

Theorem 2. Let $f: R^{2} \rightarrow R$ be a smooth function whose gradient has an isolated singularity at 0 . Then $I(\operatorname{grad} f) \leqq 1 .^{2}$

The proof runs as follows. Let $D^{2}$ be the unit disk. After a slight perturbation of $f$, the only points where $\operatorname{grad} f$ is orthogonal to the unit circle $S^{1}=\partial D^{2}$ are one of the four types in Fig. 3.

Note that the points of type I and III represent maxima of $f \mid S^{1}$, while the points of type II and IV represent minima of $f \mid S^{1}$.

2 The analogue of this statement for maps $R^{n} \rightarrow R$ with $n>2$ seems to be false. One might nevertheless ask whether this is in any way connected to the Guth-Weinberg theorem as explained by L. O'Raifeartaigh, in Lett. al Nuovo Cimento, Vol. 18, No. 7, p. 205 (1977) 


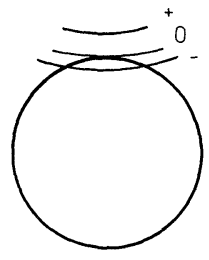

Type I

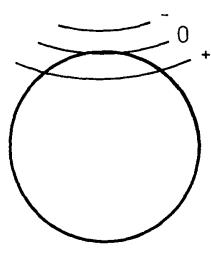

Type II

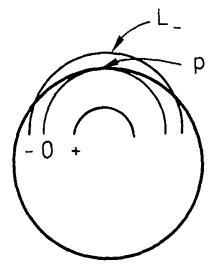

Type III

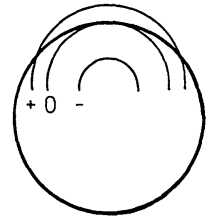

Type IV

Fig. 3. Our convention is $f($ level -$)<f($ level 0$)<f($ level +$)$

We will introduce the following notations:

$$
\begin{gathered}
\mu=\{\text { the number of points of type II }\} \\
\sigma=\{\text { the number of points of type III }\} .
\end{gathered}
$$

We have the following:

Lemma If $I(\operatorname{grad} f) \neq+1$, then $\mu \geqq \sigma$.

Before proving this, let us show how granted the lemma, one can deduce our Theorem 2.

Given our function $f$, we can perturb it slightly, keeping it fixed in a neighborhood of $S^{1}$, into a new function $g$ which has only Morse-type singularities. Clearly:

$$
I(\operatorname{grad} f)=\sum_{x \in \operatorname{Sing} g} I_{x}(\operatorname{grad} g)
$$

Also :

$$
\sum_{x} I_{x}(\operatorname{grad} g)=m-s+M
$$

where $m$ (respectively $s$, respectively $M$ ) is the number of minima of $g$ (respectively of saddle points, respectively of maxima). By using a bit of elementary Morse theory (see for example [1] or [11]), one can see that the unit disk $D^{2}$ can be reconstructed from $(m+\mu)$ handles of index $0,(s+\sigma)$ handles of index 1 , and $M$ handles of index 2.

So, one finds that:

$$
(m+\mu)-(s+\sigma)+M=\chi\left(D^{2}\right)=1,
$$

where $\chi$ denotes the Euler-Poincaré characteristic. Hence:

$$
I(\operatorname{grad} f)=1-(\mu-\sigma),
$$

and by our lemma, either $I(\operatorname{grad} f)=1$ or $\mu-\sigma \geqq 0$, so, anyway $I(\operatorname{grad} f) \leqq 1$.

The only thing left is to prove the lemma itself. Let $p$ be a point of type III. We can assume without loss of generality that the level line $L_{-}$(Fig. 3) contains no singularity and hence that it can be continued globally, as illustrated in Fig. 4a or b. 


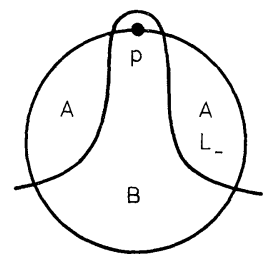

a)

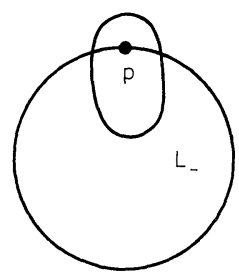

b)

Now, Fig. $4 \mathrm{~b}$ can occur only if the singularity 0 is inside $L_{-}$, and then, automatically $I(\operatorname{grad} f)=+1$.

So, if $I(\operatorname{grad} f) \neq+1$, which we assume from now on, Fig. $4 \mathrm{~b}$ is excluded.

Each $L_{-}$-level divides $D^{2}$ into a $B$-region and two $A$-regions, as in Fig. 4 a. One can introduce the following order relation in between the various type III points:

$$
\left.p_{1}<p_{2} \Leftrightarrow\left\{L_{-}\left(p_{2}\right) \subset \text { (one of the } A \text {-regions of } p_{1}\right)\right\} \text {. }
$$

Let us call "free" an $A$-region not containing another $L_{-}(p)$ level, with $p$ of type III.

The following facts are easily established:

1) Any free $A$-region not containing the origin, contains at least one point of type II.

2) The number of free $A$-regions is strictly larger than $\sigma$. This follows from the fact that any tree has strictly more endpoints than branch points.

These two points imply our lemma.

\section{On the Existence of Physical Defects Which Homotopically Speaking Should not be There}

We turn now to ordered media representable by foliations which are not necessarily measured. We consider the case $n=3, k=2$. Such situations occur for instance in $[12,13]$. We will exhibit an example of a 2-dimensional foliation $\mathscr{F}$ defined in $R^{3}$ outside the region $X=\left\{x^{2}+y^{2}<1,-1<z<1\right\}$, and such that:

$\alpha)$ The field of 2-planes defined by $\mathscr{F}$ extends continuously throughout $R^{3}$.

$\beta)$ There is no 2-dimensional foliation defined in $R^{3}$ and extending $\mathscr{F}$.

Let us add right away that this example is completely standard in the theory of foliations. We recall it here just to illustrate our point about defects.

We can start by considering a 1-dimensional foliation $\mathscr{F}_{0}$, defined on the cylinder : $\Gamma=\left\{x^{2}+y^{2}=1,-1 \leqq z \leqq 1\right\}$, such that in the regions $+1 \leqq z \leqq-\frac{1}{2}$ and $\frac{1}{2}$ $\leqq z \leqq 1$, the leaves are horizontal circles, and in the region $-\frac{1}{2}<z<\frac{1}{2}$ they are infinite lines, spiraling from the bottom circle $\left(x^{2}+y^{2}=1, z=-\frac{1}{2}\right)$ to the top circle $\left(x^{2}+y^{2}+1, z=\frac{1}{2}\right)$, as in Fig. 5 .

The foliation $\mathscr{F}$ will be defined outside the cylindrical box from Fig. 5 in such a way that:

a) For $z \geqq 1$ or $z \leqq-1$, the leaves of $\mathscr{F}$ are just the horizontal planes $z=$ const.

b) In the intermediate region, the leaves of $\mathscr{F}$ touch the lateral surface of the cylindrical box along the leaves of $\mathscr{F}_{0}$. 
Fig. 5

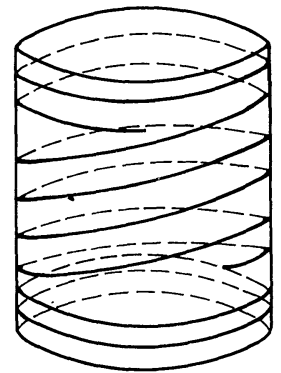

c) If $L_{0}$ is a leaf of $\mathscr{F}_{0}$, then the ruled surface defined by all the half lines ( $x=a t$, $y=b t, z=c)$, for $(a, b, c) \in L_{0}$ and $t \geqq 1$, is a leaf of $\mathscr{F}$.

This foliation $\mathscr{F}$ clearly has property $\alpha$ ).

On the other hand, by the so-called "Reeb stability theorem" [6], any foliation of the cylindrical box, which is transversal to the lateral surface and such that the lid and bottom of the box are leaves, is, after an appropriate change of coordinates, just the standard foliation by horizontal planes.

Hence, since $\mathscr{F}_{0}$ contains non-compact leaves, $\mathscr{F}$ also has property $\beta$ ).

\section{Some Comments on Defects in Gauge Fields and Crystalline Systems}

We consider now the standard mathematical model for a gauge field. This consists of the following ingredients :

a) A principal G-bundle $E \stackrel{p}{\longrightarrow} X$ where $X$ is the "physical space" and $G$ a Lie group. [At any point $x \in X$, the fiber $G_{x}=p^{-1}(x)$ is the space of some internal parameters like "phase" $(G=U(1))$ or "isospin" $(G=\mathrm{SU}(2))$ or "quark color" $(G=\mathrm{SU}(3)) \cdot]$

We assume $X$ to be an $n$-dimensional manifold.

b) A connection or "gauge potential", which associates to every $a \in E$ an $n$ dimensional plane $\Phi(a)$, tangent to $E$ at $a$, transversal to the fiber passing through $a$, depending smoothly on $a$ [and such that $\left.\Phi(g a)=\left(R_{g}\right)_{*} \Phi(a)\right]$. Thus the connection is an infinitesimal notion of horizontality on $E$, allowing us to compare infinitesimally close fibers. More exactly, if $x, y \in X$ are joined by an infinitesimal path $\varphi$, one can drag $G_{x}$ along $\varphi$, by "parallel transport" and identify it with $G_{y}$.

c) The curvature $F$ of the connection $\Phi$, or the "gauge field of the potential". This is an antisymmetric, covariant 2 nd order tensor on $X$, with values in the Lie algebra of $G$. If $x \in X$ and $\xi, \eta$ are two infinitesimal vectors at $x$, then the value $F_{\xi, \eta}(x)$ measures the infinitesimal distortion created by parallel displacement along the infinitesimal parallelogram defined by $\xi, \eta$. All these things are explained in detail in many places (see for example [2] or [14]).

The following facts are well-known:

1. The gauge field is identically zero (or in a "pure gauge" form) if and only if $\Phi$ defines a foliation of $E$, which we will denote by $\mathscr{F}_{\Phi}$. Such a connection is called flat. 
2. If we consider $G$ stripped of its topology, we have the discrete group $G_{d}$ (with the same underlying set and algebraic structure as $G$ ) and the obvious identity map $G_{d} \rightarrow G$ is continuous. The existence of a flat connection gives rise to a reduction of the structural group of $E$, from $G$ to $G_{d}$, or in terms of classifying spaces to a lift:

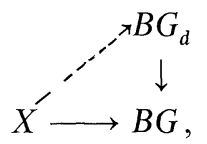

where $e$ is a map inducing our original bundle.

Conversely any such reduction gives rise to a flat connection.

3. Bundles with discrete structural group $G_{d}$ are completely characterized by their "holonomy map" (parallel transport along the leaves of $\mathscr{F}_{\Phi}$ ): $\pi_{1} X \rightarrow G_{d}$.

Of course, all these notions make sense for an open set $U \subset X$ and the restriction of $E$ to $U$. In particular, our model for defects in a gauge field will be a closed "defect set" $\Sigma \subset X$ such that on the open set $X-\Sigma$, the connection $\Phi$ is flat. The whole energy of the field is concentrated on $\Sigma$ which looks very much like a "soliton" (see $[8])$.

Let us also remark that the well-known Aharanov-Bohm effect (see for instance $[23,24])$ fits beautifully in this scheme. Namely $X$ is space-time, $E$ is the trivial $U(1)$-bundle over $X$ and $\Sigma$ is the portion of space-time where a certain magnetic field does not vanish, so that $U=X-\sum$ is homotopically like a circle. In our language, the phase shift in the Aharanov-Bohm experiment is just the holonomy of the flat bundle $E \mid U$. This bundle is of course $U(1)$-trivial, but not $U(1)_{d}$-trivial!

Our kind of defect is not to be mixed up with a Dirac monopole [23, 24], where the bundle fails to be defined on the "defect set" $\Sigma \subset X$. From a topological standpoint, such a Dirac-monopole-type of defect behaves very much like a Toulouse-Kléman defect, if the manifold of internal states is replaced by the classifying space $B G$ and the order parameter map by some map $X-\Sigma \rightarrow B G$, inducing the given bundle.

The kind of defects for a gauge field we have described before, coming from the nonflatness of the connection and not from such a simple "hole" in the bundle, are more like a "t'Hooft monopole" and are of a more subtle nature than the Dirac monopoles.

So now we can start asking a Toulouse-Kléman type of question : if $L \subset \Sigma$ is a defect pellicule of dimension $p<n$, when can we "cut" it? In more precise terms, when can we change $\Phi$ in a neighborhood of $L$, so that it becomes flat in $X-(\Sigma-L)$ ? The obvious thing to do is to surround $L$ by a $q$-dimensional sphere $S^{q}$ (with $p+q+1=n$ ) and consider the $(q+1)$-dimensional disk $\Delta^{q+1}$, spanned by $S^{q}$ and transversal to $L$ (Fig. 6).

It is convenient to thicken $\Delta^{q+1}$ to a very thin $n$-dimensional cylindrical box $\beta=\Delta^{q+1} \times[-\varepsilon, \varepsilon]^{p} C X$ and consider a very thin neighborhood of the boundary of $\Delta^{q+1}$, call it $S^{q} \times[0, \eta]$, where $S^{q} \times 0$ is $S^{q}$.

Then, the bundle $E \mid \beta$ is endowed with a flat connection on $\alpha=S^{q} \times[0, \eta] \times[-\varepsilon, \varepsilon]^{p}$ and the problem is how to extend it. There are some 
Fig. 6

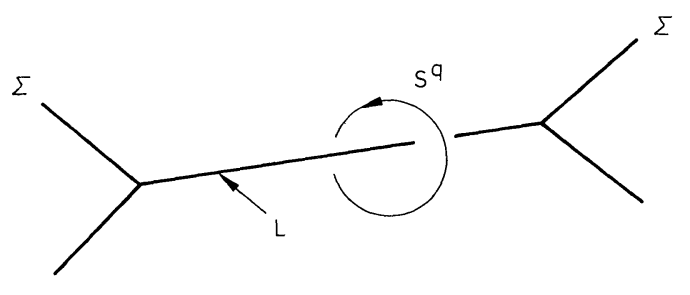

obvious necessary conditions which are listed below:

(i) If $p=n-2$ and $q=1$, then $E \mid \alpha$ possesses a holonomy map $\pi_{1} S^{1} \stackrel{h}{\longrightarrow} G$ which is characterized by the image $h(1) \in G$. In order to be able to cut $L$, one has to have $h(1)=\{$ the unit element of $G\}$.

(ii) If $p<n-2$ and $q \geqq 2$, the flat connection on $E \mid \alpha$ trivializes this bundle. The obstruction to extend this trivialization is an element:

$$
\omega \in \pi_{q}\left(B G, B G_{d}\right)=\pi_{q-1} G
$$

(we use the fact that $q \geqq 2$ ).

In order to be able to cut $L$, one has to have $\omega=0$. This kind of obstruction has also been described by Toulouse, from a different viewpoint [21].

All this is a local story "à la Toulouse-Kléman", and by globalizing it, one should be able to recapture a description of those characteristic classes which die on a flat bundle, and nothing more (see also the last section of [17]). Which also means that the complete story of how to cut defects in gauge fields has to be more complicated, involving, possibly, the homotopy groups of the classifying spaces of foliations (as defined in [4] and extensively studied in various papers of Mather and Thurston).

To conclude this section, we come to the problem of defects of crystalline systems. It is known that the standard homotopy approach can capture dislocations (the Burgers vectors), but is somewhow inappropriate for other defects, like disclinations, where rotational symmetry is also involved. We want to suggest that the preceding approach could again be useful here.

Consider $E=\left\{\right.$ the bundle of all orthogonal 3-frames in $\left.R^{3}\right\}$. This is a principal $\mathrm{SO}(3)$-bundle. Notice that in the region of space $U \subset R^{3}$ where the crystal is undistorted, it naturally defines a flat connection in this principal bundle. (The crystalline system gives a precise rule of how to move a frame from $x$ to $y$; it is easy to see that this is a flat connection.) Disclinations clearly correspond to regions of nonzero curvature, except that curvature here is more like a "Dirac function".

But such Dirac-type curvatures, which are already familiar objects, occur in other areas of research, too (see for instance [18]). We plan to come back to all these things in a future paper.

\section{Appendix : Foliations}

To simplify matters, we consider only foliations of dimension $n-1$ on $n$-manifolds.

Locally at least, a field of $(n-1)$-tangent planes to $M^{n}$, can be given by a nonzero differential form: $\omega=\Sigma a_{i}(x) d x^{i}$, which vanishes exactly along the direc- 
tions contained in the field $\Phi$. The field $\Phi$ defines a foliation $\mathscr{F}$ if and only if the following condition is fulfilled: $\omega \wedge d \omega=0$.

This is a non-linear equation called the "Frobenius integrability condition".

Moreover, $\Phi$ defines a "measured foliation", which means a foliation where "leaves stay at equal distance" if and only if the following stronger linear condition is fulfilled:

$$
d \omega=0
$$

A good reference for the general theory of foliations is [6]. For measured foliations in the two-dimensional case, one can consult [19].

Acknowledgements. We are indebted to several colleagues for advice and dicussion. In particular we want to thank F. Axel, S. Ciulli, P. Ginsparg, J. Fröhlich, F. Laudenbach, L, Michel, V. P. Mineev, S. Sidhastrou, G. Toulouse, and G. E. Volovik.

\section{References}

1. Bott, R.: Lectures on Morse theory. Bonn (1960)

2. Daniel, M., Viallet, C.M.: Rev. Mod. Phys. 52, 175-197 (1980)

3. Flanders, H.: Differential forms with applications to the physical sciences. London, New York: Academic Press 1963

4. Haefliger, A.: Homotopy and integrability, in manifolds, Amsterdam 1970. In: Lecture Notes in Mathematics, Vol. 197, pp. 133-163. Berlin, Heidelberg, New York: Springer 1971

5. Langevin, R.: Feuilletages et cristaux liquides (to appear)

6. Lawson, B.: The quantitative theory of foliations. Conference board of AMS, No. 27 (1975)

7. Kléman, M., Michel, L., Toulouse, G. : J. Phys. Lett. 38, 195 (1977)

8. Madore, J.: Geometrical methods in classical field theory (to appear)

9. Mermin, N.D.: Rev. Mod. Phys. 51, 591-648 (1979)

10. Michel, L.: Rev. Mod. Phys. 52, 617-651 (1980)

11. Milnor, J.: Morse theory. Princeton: Princeton University Press 1963

12. Mineyev, V.P., Volovik, G.E. : Planar and linear solitons in superfluid ${ }^{3} \mathrm{He}$ (preprint)

13. Mineyev, V.P.: On the classification of topologically stable singularities in cholesteric liquid crystals and in other ordered media with non homogeneous ground state (preprint)

14. Nomizu, K.: Lie groups and differential geometry. Math. Soc. of Japan (1956)

15. Poénaru, V., Toulouse, G.: J. Physique 887, 887-895 (1977)

16. Poénaru, V., Toulouse, G.: J. Math. Phys. 20, 13-19 (1979)

17. Poénaru, V.: Elementary algebraic topology related to the theory of defects and textures, in Illcondensed matter. Les Houches 1978, pp. 265-318 (1979)

18. Poénaru, V.: On ergodic theory, in Ill-condensed matter. Les Houches 1978, pp. 565-607 (1979)

19. Fathi, A., Laudenbach, F., Poénaru, V.: Travaux de Thurston sur les surfaces. Astérisque 66-67 (1979)

20. Toulouse, G., Kléman, M.: J. Phys. 37, 149-151 (1976)

21. Toulouse, G.: Gauge concepts in condensed matter physics. Cargèse 1979 (to appear)

22. Volovik, G.E., Mineyev, V.P.: Investigations of singularities in superfluid $\mathrm{He}^{3}$ in liquid crystals by the homotopic topology methods. J.E.T.P. 45 (1977)

23. Yang, G.N.: Phys. Rev. Lett. 33, 445-447 (1974)

24. Wu, T.T., Yang, C.N.: Phys. Rev. D 12, 3845-3857 (1975)

Communicated by A. Jaffe

Received December 17, 1980 